\title{
SÍNDROME DE EFUSIÓN UVEAL SECUNDARIO A CIRUGÍA DE ESTRABISMO
}

\section{UVEAL EFFUSION SYNDROME AFTER STRABISMUS SURGERY}

\author{
MERINO P ${ }^{1}$, GÓMEZ DE LIAÑO P², YÁÑEZ MARTÍNEZ J²
}

\begin{abstract}
RESUMEN
Caso clínico: Descripción de una mujer operada de estrabismo con anestesia tópica, con conjuntivitis exudativa en el postoperatorio inmediato, que a los 15 días tuvo un síndrome de efusión uveal. El tratamiento fue la exploración quirúrgica, retirada de las suturas de vycril, antibioterapia y corticoterapia tópica, subtenoniana y sistémica, aislándose Staphylococcus epidermidis en las muestras intraoperatorias. Evolucionó favorablemente en 4 meses. Discusión: La efusión uveal secundaria a cirugía de estrabismo no está publicada en la literatura revisada. La infección de las suturas en la cirugía escleral de retina por Staphylococcus epidermidis se considera una posible, aunque rara, causa.
\end{abstract}

Palabras clave: Síndrome de efusión uveal, cirugía de estrabismo, escleritis, Staphylococcus epidermidis, infección de suturas.

\begin{abstract}
Case report: A woman was operated on for strabismus under topical anesthesia. An exudative conjunctivitis was diagnosed immediately after the surgery, and a uveal effusion syndrome diagnosed 15 days later. Surgery exploration, vycril suture removal and therapy with systemic, sub-tenon, and topical corticosteroid and antibiotics was initiated. Intraoperative culture revealed evidence of Staphylococcus epidermidis. Good resolution occurred in 4 months.

Discussion: Uveal effusion secondary to strabismus surgery has not been reported in the published literature. Implant and suture infection by Staphylococcus epidermidis following scleral buckling surgery may be a possible, but rare, cause of the uveal effusion syndrome (Arch Soc Esp Oftalmol 2006; 81: 409-412).
\end{abstract}

Key words: Uveal effusion syndrome, strabismus surgery, scleritis, Staphylococcus epidermidis, suture infection.

\section{INTRODUCCIÓN}

El síndrome de efusión uveal se produce por una exudación anormal de fluido seroso a partir de la coriocapilar, que se acumula en la coroides y el espacio subretiniano. Entre las causas que provocan este cuadro se han descrito factores inflamatorios, hidrodinámicos, osmóticos e idiopáticos $(1,2)$. La etiología inflamatoria comprende: la escleritis, uveítis, cirugía y traumatismo intraocular, infección del

\footnotetext{
Recibido: 16/9/05. Aceptado: 19/7/06.

Sección de Motilidad Ocular. Departamento de Oftalmología. HGU Gregorio Marañón. Madrid. España.

1 Doctora en Medicina.

2 Licenciado en Medicina.

Correspondencia:

Pilar Merino Sanz

C/. Marquéz de Lozoya, 14, Esc. 1, 14-B

28007 Madrid

España

E-mail: jacoboym@telefonica.net
} 
cerclaje o explante escleral, la fotocoagulación y la criocoagulación (1). La escleritis primaria o secundaria a una infección de un cerclaje o sutura escleral provoca una inflamación de la coroides con vasodilatación y efusión de líquido seroso en la coroides y espacio subretiniano. Clínicamente se desarrolla un desprendimiento coroideo (DC) periférico de morfología anular, que deja ver la ora serrata sin indentar, así como un desprendimiento de retina (DR) bulloso, que se desplaza con los movimientos cefálicos y oculares; también se observa dilatación de vasos epiesclerales, la PIO es normal, células escasas en vítreo e hiperplasia multifocal del EPR originando «manchas de leopardo». El tratamiento consiste en la retirada de las suturas y material plástico infectado, y la administración de antibióticos y corticoides tópicos y sistémicos (1).

\section{CASO CLÍNICO}

Presentamos el caso de una mujer de 76 años, diabética, que fue operada de estrabismo con una retroinserción ajustable de recto inferior de OI con anestesia tópica, por presentar diplopía al día siguiente de la cirugía de cataratas. Su AV inicial era de 0,7 . Era emétrope, y la longitud axial del globo ocular se encontraba dentro de los límites normales. En el postoperatorio inmediato se observó una conjuntivitis exudativa refractaria al tratamiento tópico con ciprofloxacino y tobramicina. A los 15 días la paciente acudió de urgencias por dolor ocular intenso, observándose MOE normal, edema de párpado inferior, dilatación de vasos epiesclerales, PIO de $15 \mathrm{mmHg}$. En el fondo de ojo presentaba un DC inferior que en 24 horas evolucionó a anular, apreciándose ora serrata sin indentación. La enferma fue ingresada para realizar estudio y tratamiento. A las 48 horas refirió pérdida de visión, la AV era de percepción de luz, observándose un DR exudativo total (figs. 1 y 2). La analítica era normal. Se realizó cultivo de exudado conjuntival, y ecografía ocular donde se apreciaba engrosamiento escleral a nivel del recto inferior, sin signos de abceso. En el TAC se confirmó el diagnóstico de desprendimiento coroideo seroso, sin signos hemorrágicos, y de escleritis (fig. 3). Se instauró tratamiento con levofloxacino y prednisona orales. El cultivo del exudado resultó estéril por lo que se continuó con el mismo tratamiento tópico de norfloxacina, metilprednisolona y ciclopentolato. Ante la mala evolu-

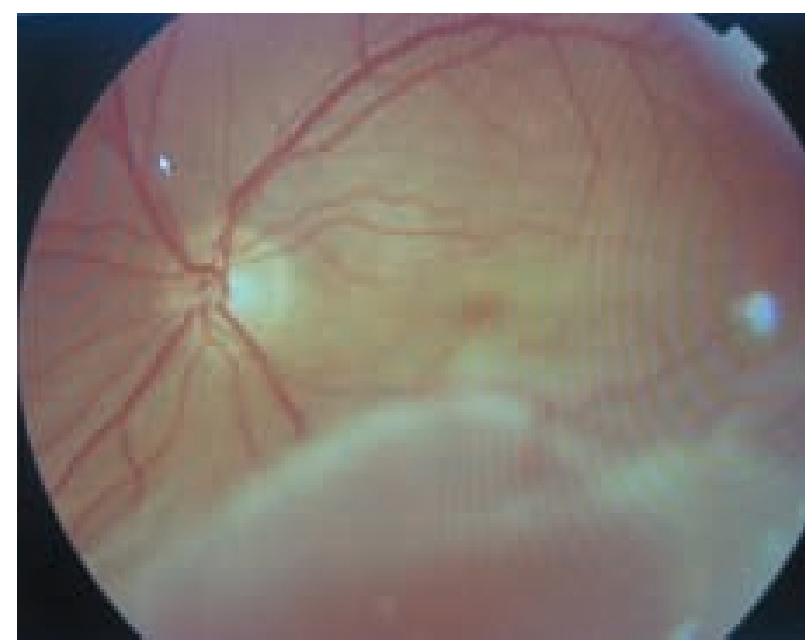

Fig. 1: DR exudativo en resolución que afecta la mácula.

ción del cuadro, al $5 .^{\circ}$ día se procedió a la exploración quirúrgica del recto inferior y a la retirada de las suturas musculares a esclera de vycril 6/0. El músculo presentaba buena coloración, pero había una gran adherencia a la esclera subyacente, la cual tenía una textura algo rugosa, de color crema. Se inyectó vancomicina y dexametasona subtenonianas. El cultivo de las muestras conjuntivales intraoperatorias fue positivo a Staphylococcus epidermidis sensible a aureomicina y vancomicina, por lo que se pautó aureomicina tópica. Se mantuvo el tratamiento de levofloxacino oral durante 15 días y la prednisona oral fue disminuyéndose gradualmente cuando se reaplicó la retina, el ciclopentolato y la metilprednisolona tópicas se continuaron hasta que se resolvió el DC. El desprendimiento de retina se fue resolviendo hasta solucionarse totalmente en 75 días (asociando tratamiento postural para evitar el desprendimiento macular), el DC en 105 días, quedando con una AV final de 0,7, y unas alteraciones pigmentarias multifocales en toda la periferia retiniana. No se observaba ninguna cicatriz, ni marca coriorretiniana a nivel de la inserción primitiva del recto inferior que indicaran punción inadvertida en la cirugía de estrabismo.

\section{DISCUSIÓN}

La diferencia clínica fundamental entre la efusión uveal primaria o idiopática y la secundaria a una escleritis posterior es la ausencia de dolor en el primer caso, mientras que la pérdida de visión se 


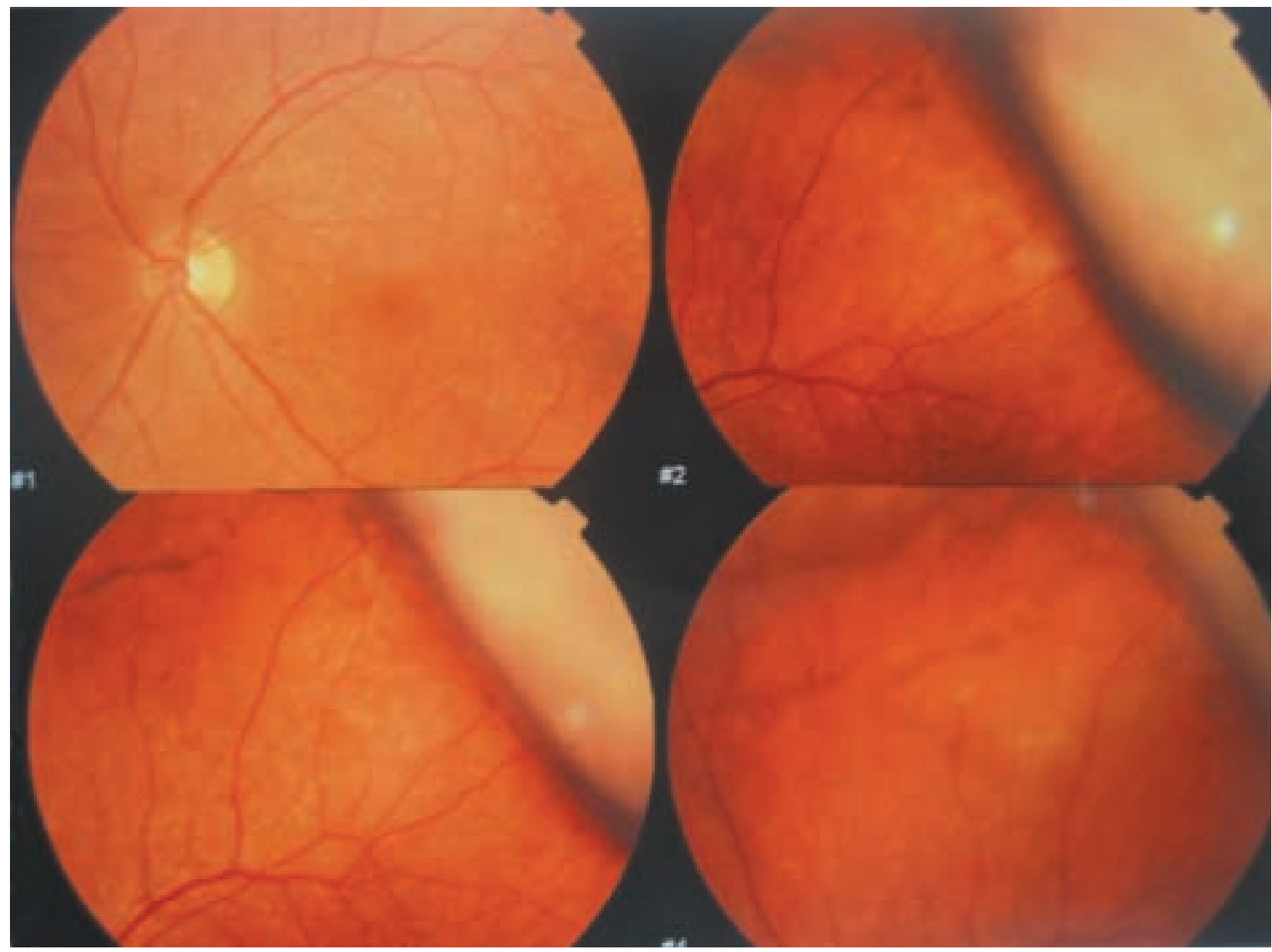

Fig. 2: Desprendimiento coroideo (DC) seroso.

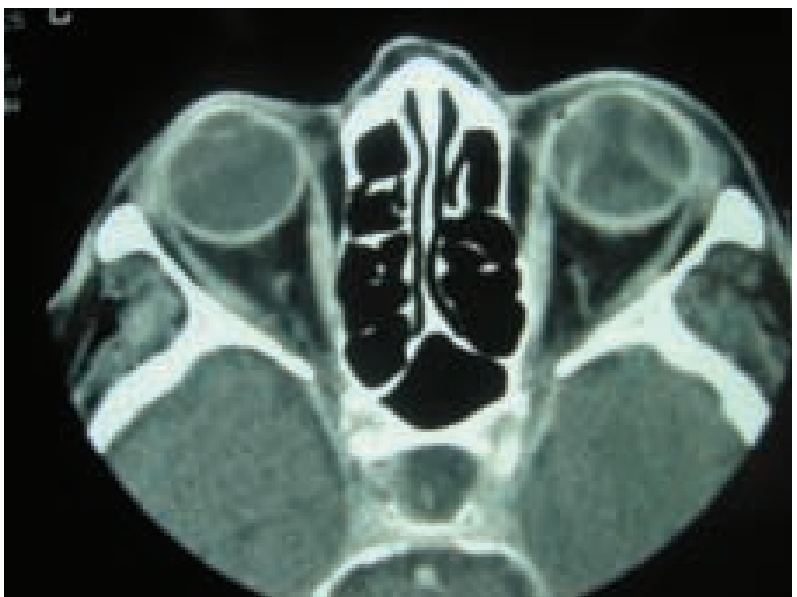

Fig. 3: En el TAC del OI se aprecia DR y DC, con engrosamiento de la esclera posterior. produce gravemente en ambas debido al DR exudativo que afecta a la mácula $(3,4)$.

La revisión de la literatura realizada por nosotros no ha encontrado ninguna referencia a la asociación de cirugía de estrabismo con el típico síndrome de efusión uveal, aunque la incidencia de daño coroideo sin perforación es del 0,6\% según algunos autores, y la de perforación varía del $0,13 \%$ al $12 \%$ (5). En este caso los antecedentes de la cirugía, la conjuntivitis exudativa postoperatoria, el dolor ocular, la pérdida de visión, los hallazgos oftalmoscópicos y las pruebas de imagen confirman el diagnóstico de síndrome de efusión uveal secundario a escleritis posterior, descartándose la perforación coriorretiniana por no encontrarse signos clínicos oftalmoscópicos que la puedan confirmar. 
El diagnóstico diferencial habría que hacerlo entre la etiología infecciosa de la escleritis y la reacción inflamatoria a las suturas de vycril $(1,4)$. A favor de la infección estaría el cultivo positivo a Staphylococcus epidermidis de las muestras de tejido intraoperatorio (1), aunque para otros autores se trate de un germen no patógeno que no requiere tratamiento antibiótico (4).

La realización de la cirugía de estrabismo con anestesia tópica podría hipotéticamente contribuir a una mayor contaminación del campo quirúrgico al tener que incorporar al paciente en la camilla para comprobar la desviación ocular. Sin embargo, en la actualidad no hay estudios que puedan aportar ningún dato que apoye esta hipótesis.

En nuestro caso la retirada de las suturas esclerales de vycril y la administración tópica, subtenoniana y sistémica de antibióticos y corticoides pudo resolver el cuadro recuperándose la AV inicial, lo que apoyaría, en nuestra opinión, la etiología infecciosa de la escleritis. El carácter exudativo del des- prendimiento retiniano que se modificaba con los movimientos cefálicos facilitó que la mácula no estuviera desprendida el tiempo suficiente para alterar irreversiblemente la visión.

\section{BIBLIOGRAFÍA}

1. Brockhurst RJ. Ciliochoroidal (uveal) efusión. In: Ryan SJ. Retina. St. Louis: Mosby; 1994; II: 1745-1752.

2. Uyama M, Takahashi K, Kozaki J, Tagami M, Takada Y, Ohkuma $H$, et al. Uveal effusion syndrome: clinical features, surgical treatment, histologic examination of the sclera, and pathophysiology. Ophthalmology 2000; 107: 441-449.

3. Leitch RJ, Bearn MA, Watson PG. Exudative retinal detachment and posterior scleritis associated with massive scleral thickening and calcification treated by scleral decompression. Br J Ophthalmol 1992; 76: 109-112.

4. Topilow HW, Ackerman AL. Massive exudative retinal and choroidal detachments following scleral buckling surgery. Ophthalmology 1983; 90: 143-147.

5. Noel LP, Boom JN, Clarke WN, Bawazeer A. Retinal perforation in strabismus surgery. J Pediatr Ophthalmol Strabismus 1997; 34: 115-117. 